\title{
Billion Cells
}

National Cancer Institute

\section{Source}

National Cancer Institute. Billion Cells. NCI Thesaurus. Code C67247.

A unit of cell count expressed in billions. 